\title{
NONLINEAR DYNAMIC ANALYSIS OF PLATES STIFFENED BY PARALLEL BEAMS WITH DEFORMABLE CONNECTION
}

\author{
J. A. Dourakopoulos ${ }^{1}$ and E. J. Sapountzakis ${ }^{2}$ \\ ${ }^{1}$ School of Civil Engineering, National Technical University \\ Zografou Campus, GR-157 80 Athens, Greece \\ e-mail: jdour@mail.ntua.gr \\ 2e-mail: cvsapoun@central.ntua.gr
}

Keywords: Elastic stiffened plate, plate reinforced with beams, nonlinear dynamic analysis, slab-and-beam structure, deformable connection.

\begin{abstract}
In this paper a general solution to the geometrically nonlinear dynamic analysis of plates stiffened by arbitrarily placed parallel beams of arbitrary doubly symmetric cross section, subjected to arbitrary dynamic loading is presented. The plate-beam structure is assumed to undergo moderate large deflections and the nonlinear analysis is carried out by retaining nonlinear terms in the kinematical relations. According to the proposed model, the arbitrarily placed parallel stiffening beams are isolated from the plate by sections in the lower outer surface of the plate, making the hypothesis that the plate and the beams can slip in all directions of the connection without separation (i.e. uplift neglected) and taking into account the arising tractions in all directions at the fictitious interfaces. These tractions are integrated with respect to each half of the interface width resulting in two interface lines, along which the loading of the beams as well as the additional loading of the plate is defined. Their unknown distribution is established by applying continuity conditions in all directions at the interfaces taking into account their relation with the interface slip through the shear connector stiffness. The utilization of two interface lines for each beam enables the nonuniform distribution of the interface transverse shear forces and the nonuniform torsional response of the beams to be taken into account describing better in this way the actual response of the platebeams system. Six boundary value problems are formulated and solved using the Analog Equation Method (AEM), a BEM-based method. Application of the boundary element technique leads to a nonlinear coupled system of equations of motion, which is solved employing a distributed mass model. Both free and forced transverse vibrations are considered and numerical examples with great practical interest are presented demonstrating the effectiveness, wherever possible the accuracy and the range of applications of the proposed method. The adopted model permits the evaluation of the shear forces at the interfaces in both directions, the knowledge of which is very important in the design of prefabricated ribbed plates.
\end{abstract}




\section{INTRODUCTION}

Stiffened plate panels are structural elements of practical importance in applications such as ship superstructures, bridge decks and aircraft structures. Stiffening of the plate provides the benefit of added load-carrying capability with a relatively small additional weight penalty, while buckling is prevented especially in case of in-plane loading. The unique peculiarities of the aforementioned structures are obtained due to the behavior of the bond between the plate and the beams; however this bond is the usual reason why these structures are prone to failure. Moreover, since these stiffened plates are frequently subjected to dynamic loading such as air blasts or underwater explosions, a clear understanding of the dynamic response requires development of an efficient dynamic analysis capability.

When the deflections of the structure are small, a wide range of linear analysis tools, such as modal analysis, can be used and some analytical results are possible. As the deflections become larger, the induced geometric nonlinearities result in effects that are not observed in linear systems, making therefore the determination of an analytical solution extremely difficult and in most cases impossible. Moreover, having in mind the importance of weight saving in engineering structures, the study of nonlinear effects in the analysis (large deflection analysis) of stiffened plates becomes essential. These nonlinearities result from retaining the squares of the slopes in the strain-displacement relations (intermediate nonlinear theory), avoiding in this way the inaccuracies arising from a linear or a linearized second-order analysis.

The behavior of composite slab-and-beam structure is affected significantly by the deformability of the shear connection between plate and beams. Therefore, much research effort has been done concerning the linear dynamic analysis of composite beams with deformable connections. On the other hand, less research effort has been made on the free or forced dynamic analysis, taking also account the geometric nonlinearities.

In this paper, a general solution to the geometrically nonlinear dynamic analysis of plates stiffened by arbitrarily placed parallel beams of arbitrary doubly symmetric cross section with deformable connections subjected to arbitrary dynamic loading is presented. The solution is based on the structural model proposed by Sapountzakis and Mokos in [1], according to which the stiffening beams are isolated from the plate by sections in the lower outer surface of the plate, making the hypothesis that the plate and the beams can slip in all directions of the connection without separation (i.e. uplift is neglected) and taking into account the arising tractions in all directions at the fictitious interfaces. These tractions are integrated with respect to each half of the interface width resulting in two interface lines, along which the loading of the beams as well as the additional loading of the plate is defined. The unknown distribution of the aforementioned integrated tractions is established by applying continuity conditions in all directions at the two interface lines taking into account their relationship with the interface slip through the shear connector stiffness. Any distribution of connectors in each direction of the interfaces can be handled. The utilization of two interface lines for each beam enables the nonuniform torsional response of the beams to be taken into account as the angle of twist is indirectly equated with the corresponding plate slope. Six boundary value problems are formulated and solved using the Analog Equation Method (AEM) [2], a BEM based method.

The essential features and novel aspects of the present formulation are summarized as follows.

- The adopted model permits the evaluation of the longitudinal and transverse in-plane shear forces at the interfaces between the plate and the beams in the geometrically nonlinear dynamic analysis of the stiffened plate, taking into account the influence of interface slip, the knowledge of which is very important in the design of shear connectors in plate-beam structures. 
- Utilization of two interface lines permits the nonuniform distribution of the in-plane forces along the interface width to be taken into account.

- Both free and forced transverse vibrations are considered taking into account geometric nonlinearities.

- The stiffened plate is of arbitrary shape and is subjected to arbitrary dynamic loading, while both the number and the placement of the parallel beams are also arbitrary (eccentric beams are included).

- The cross section of the stiffening beams is an arbitrary doubly symmetric thin or thick-walled one. The formulation does not stand on the assumption of a thin-walled structure and therefore the cross section's torsional and warping rigidities are evaluated "exactly" in a numerical sense employing the BEM (requiring only boundary discretization for the cross sectional analysis).

- The plate and the beams are supported by the most general boundary conditions including elastic support or restraint.

- The nonuniform torsion in which the stiffening beams are subjected is taken into account by solving the corresponding problem and by comprehending the arising twisting and warping in the corresponding displacement continuity conditions. The distributed warping moment arising from the nonuniform distribution of longitudinal in-plane forces is also taken into account.

- Contrary to previous research efforts where the numerical analysis is based on BEM using a lumped mass assumption model after evaluating the flexibility matrix at the mass nodal points, in this work a distributed mass model is employed.

Based on the numerical solution developed, several examples with great practical interest are presented, demonstrating the effectiveness, wherever possible the accuracy and the range of applications of the proposed method.

\section{STATEMENT OF THE PROBLEM}

Let us consider a thin plate of homogeneous, isotropic and linearly elastic material with modulus of elasticity $E_{p}$, volume mass density $\rho_{p}$, shear modulus $G_{p}$ and Poisson's ratio $v_{p}$, having constant thickness $h_{p}$ and occupying the two-dimensional multiply connected region $\Omega$ of the $x y$ plane bounded by the piecewise $\Gamma_{j} \quad(j=0,1,2, \ldots, K)$ boundary curves. The plate is stiffened by a set of $i=1,2, \ldots, I$ arbitrarily placed parallel beams of arbitrary doubly symmetric cross section of area $A_{b}^{i}$ and length $l^{i}$. The material of the beams is considered to be homogeneous, isotropic and linearly elastic with modulus of elasticity $E_{b}^{i}$, mass density $\rho_{b}^{i}$, shear modulus $G_{b}^{i}$ and Poisson's ratio $v_{b}^{i}$. For the sake of convenience the $x$ axis is taken parallel to the beams of length $l^{i}$ which may have either internal or boundary point supports. The stiffened plate is subjected to the arbitrary lateral dynamic load, $g=g(\mathbf{x}, t), \mathbf{x}:\{x, y\}, t \geq 0$. Owing to this loading, the plate and the beams can slip in all directions of the connection without separation (i.e. uplift is neglected). For the analysis of the aforementioned problem a global coordinate system $O x y$ for the analysis of the plate and local coordinate ones $C^{i} x^{i} y^{i}$ corresponding to the centroid axes of each beam are employed (Figure 1). 


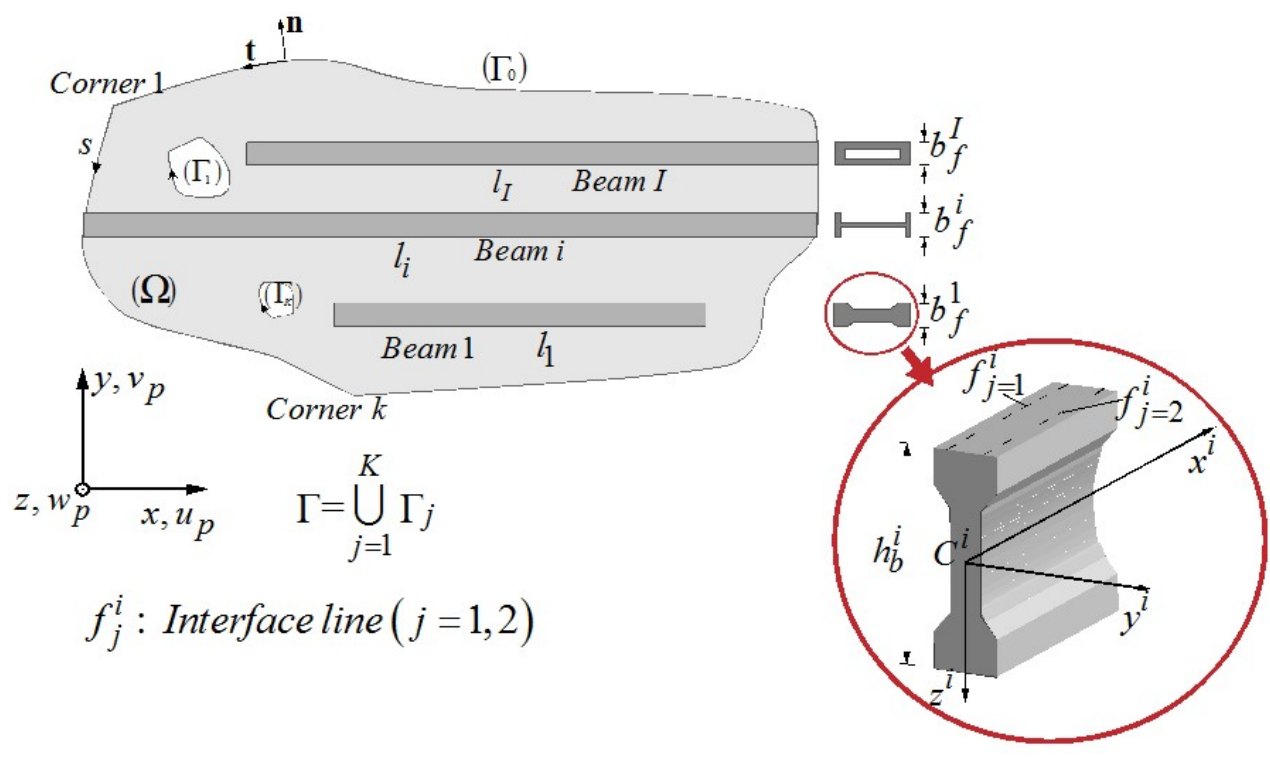

Figure 1: Two dimensional region $\Omega$ occupied by the plate.

The solution of the problem at hand is approached employing the improved model proposed by Sapountzakis and Mokos in [1]. According to this model, the stiffening beams are isolated from the plate by sections in its lower outer surface, taking into account the arising tractions at the fictitious interfaces (Figure 2). Integration of these tractions along each half of the width of the i-th beam results in line forces per unit length in all directions in two interface lines, which are denoted by $q_{x j}^{i}, q_{y j}^{i}$ and $q_{z j}^{i}(j=1,2)$ encountering in this way the nonuniform distribution of the interface transverse shear forces $q_{y}^{i}$, which in previous models [3] was ignored. The aforementioned integrated tractions result in the loading of the $\mathrm{i}$-th beam as well as the additional loading of the plate. Their distribution is unknown and can be established by imposing displacement continuity conditions in all directions along the two interface lines, enabling in this way the nonuniform torsional response of the beams to be taken into account. The arising additional loading at the middle surface of the plate and the loading along the centroid of each beam (Figure 3) can be summarized as follows:

\section{a. In the plate (at the traces of the two interface lines $j=1,2$ of the $i$-th plate-beam interface)}

(i) An in-plane line body force $q_{x j}^{i}$ at the middle surface of the plate.

(ii) An in-plane line body force $q_{y j}^{i}$ at the middle surface of the plate.

(iii) A lateral line load $q_{z j}^{i}$.

(iv) A lateral line load $\partial m_{p y j}^{i} / \partial x$ due to the eccentricity of the component $q_{x j}^{i}$ from the middle surface of the plate. $m_{p y j}^{i}=q_{x j}^{i} h_{p} / 2$ is the bending moment.

(v) A lateral line load $\partial m_{p x j}^{i} / \partial y$ due to the eccentricity of the component $q_{y j}^{i}$ from the middle surface of the plate. $m_{p x j}^{i}=q_{y j}^{i} h_{p} / 2$ is the bending moment. 


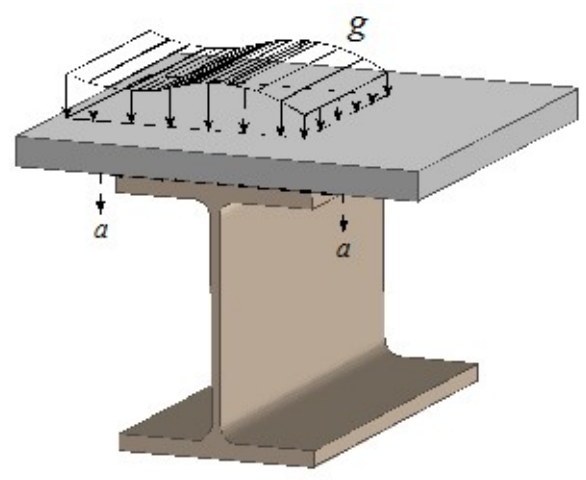

(a)

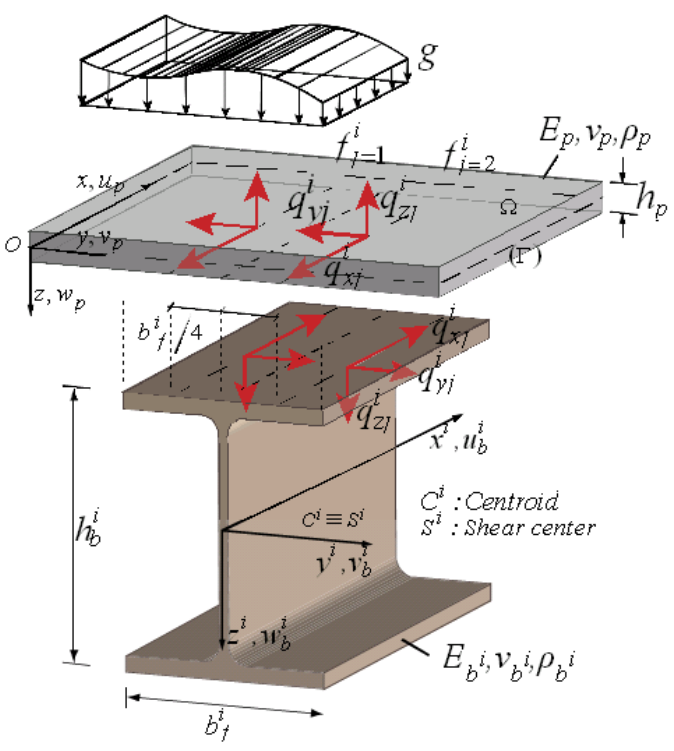

Figure 2: Thin elastic plate stiffened by beams (a) and isolation of the beams from the plate (b).

\section{b. In each (i-th) beam ( $C^{\mathrm{i}} \mathrm{x}^{\mathrm{i}} \mathrm{y}^{\mathrm{i}} \mathrm{z}^{\mathrm{i}}$ system of axes)}

(i) An axially distributed line load $q_{x j}^{i}$ along the beam centroid axis $C^{i} x^{i}$.

(ii) A transversely distributed line load $q_{y j}^{i}$ along the beam centroid axis $C^{i} x^{i}$.

(iii) A perpendicularly distributed line load $q_{z j}^{i}$ along the beam centroid axis $C^{i} x^{i}$.

(iv) A distributed bending moment $m_{b y j}^{i}=q_{x j}^{i} e_{z j}^{i}$ along $C^{i} y^{i}$ local beam centroid axis due to the eccentricities $e_{z j}^{i}$ of the components $q_{x j}^{i}$ from the beam centroid axis. $e_{z 1}^{i}=e_{z 2}^{i}=-h_{b}^{i} / 2$ are the eccentricities.

(v) A distributed bending moment $m_{b z j}^{i}=-q_{x j}^{i} e_{y j}^{i}$ along $C^{i} z^{i}$ local beam centroid axis due to the eccentricities $e_{y j}^{i}$ of the components $q_{x j}^{i}$ from the beam centroid axis. $e_{y 1}^{i}=-b_{f}^{i} / 4, e_{y 2}^{i}=b_{f}^{i} / 4$ are the eccentricities.

(vi) A distributed twisting moment $m_{b x j}^{i}=q_{z j}^{i} e_{y j}^{i}-q_{y j}^{i} e_{z j}^{i}$ along $C^{i} x^{i}$ local beam shear center axis due to the eccentricities $e_{z j}^{i}$, $e_{y j}^{i}$ of the components $q_{y j}^{i}, q_{z j}^{i}$ from the beam shear center axis, respectively. $e_{z 1}^{i}=e_{z 2}^{i}=-h_{b}^{i} / 2$ and $e_{y 1}^{i}=-b_{f}^{i} / 4$, $e_{y 2}^{i}=b_{f}^{i} / 4$ are the eccentricities.

(vii) A distributed warping moment $m_{b w j}^{i}=-q_{x j}^{i}\left(\varphi_{S}^{P i}\right)_{f j}$ along $C^{i} x^{i}$ local beam shear center axis, which in previous models $[1,3]$ was ignored. $\left(\varphi_{S}^{P i}\right)_{f j}$ is the value of the 
primary warping function $\varphi_{S}^{P i}$ with respect to the shear center of the beam cross section (coinciding with its centroid) at the point of the j-th interface line of the i-th plate - beam interface.

On the base of the above considerations the response of the plate and the beams may be described by the following boundary value problems.

\section{a. For the plate}

The analysis of the plate is based on the Von Kármán plate theory, according to which the deflection of the plate cannot be regarded as small as compared to the plate thickness, while it remains small in comparison with the rest dimensions of the plate. Due to this assumption, geometrical nonlinearities should be taken into account and the displacement field of an arbitrary point of the plate, as implied by the Kirchhoff hypothesis, is given as

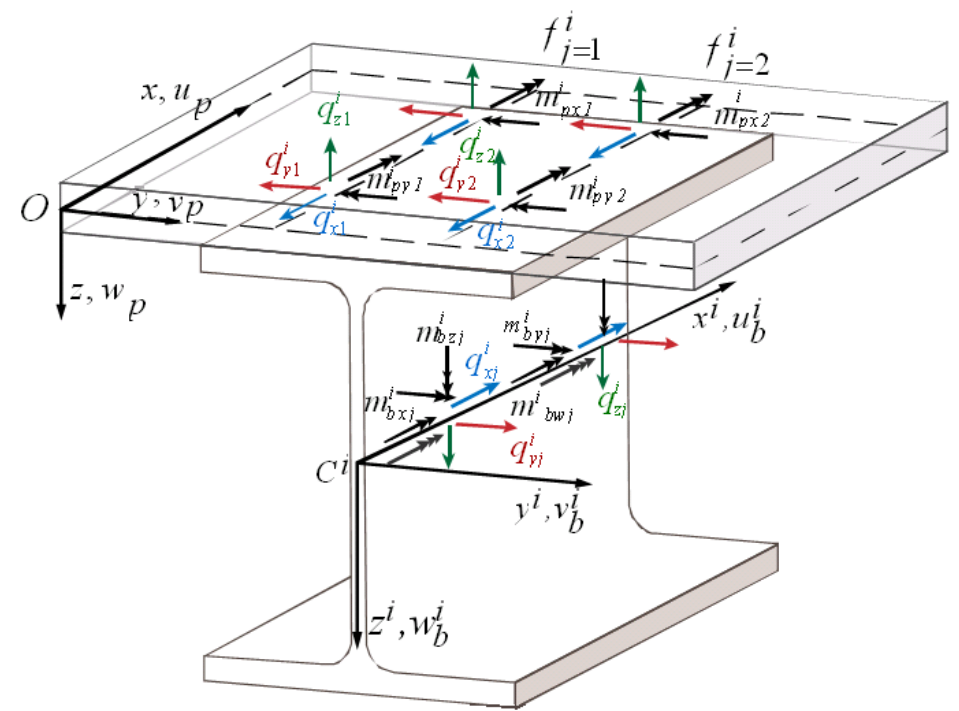

Figure 3: Structural model and directions of the additional loading of the plate and the i-th beam.

$$
\begin{gathered}
\bar{u}_{p}(x, y, z, t)=u_{p}(x, y, t)-z \frac{\partial w_{p}(x, y, t)}{\partial x} \\
\bar{v}_{p}(x, y, z, t)=v_{p}(x, y, t)-z \frac{\partial w_{p}(x, y, t)}{\partial y} \\
\bar{w}_{p}(x, y, z, t)=w_{p}(x, y, t)
\end{gathered}
$$

where $\bar{u}_{p}, \bar{v}_{p}, \bar{w}_{p}, t \geq 0$ are the time dependent in-plane and transverse displacement components of an arbitrary point of the plate and $u_{p}=u_{p}(\mathbf{x}, t), v_{p}=v_{p}(\mathbf{x}, t)$ and $w_{p}=w_{p}(\mathbf{x}, t) \mathbf{x}:\{x, y\}, t \geq 0$ are the corresponding components of a point at its middle surface. Employing the strain-displacement relations of the three-dimensional elasticity for moderate large displacements and the stress-strain relations defined by the Hooke's law, the nonvanishing components of the second Piola - Kirchhoff stress tensor are obtained as 


$$
\begin{gathered}
S_{x x}=\frac{E_{p}}{\left(1-v_{p}^{2}\right)}\left\{\left[\frac{\partial u_{p}}{\partial x}-z \frac{\partial^{2} w_{p}}{\partial x^{2}}+\frac{1}{2}\left(\frac{\partial w_{p}}{\partial x}\right)^{2}\right]+v_{p}\left[\frac{\partial v_{p}}{\partial y}-z \frac{\partial^{2} w_{p}}{\partial y^{2}}+\frac{1}{2}\left(\frac{\partial w_{p}}{\partial y}\right)^{2}\right]\right\} \\
S_{y y}=\frac{E_{p}}{\left(1-v_{p}^{2}\right)}\left\{v_{p}\left[\frac{\partial u_{p}}{\partial x}-z \frac{\partial^{2} w_{p}}{\partial x^{2}}+\frac{1}{2}\left(\frac{\partial w_{p}}{\partial x}\right)^{2}\right]+\left[\frac{\partial v_{p}}{\partial y}-z \frac{\partial^{2} w_{p}}{\partial y^{2}}+\frac{1}{2}\left(\frac{\partial w_{p}}{\partial y}\right)^{2}\right]\right\} \\
S_{x y}=\frac{E_{p}}{2\left(1+v_{p}\right)}\left(\frac{\partial u_{p}}{\partial y}+\frac{\partial v_{p}}{\partial x}-2 z \frac{\partial^{2} w_{p}}{\partial x \partial y}+\frac{\partial w_{p}}{\partial x} \frac{\partial w_{p}}{\partial y}\right)
\end{gathered}
$$

Subsequently, integrating the stress components over the plate thickness, the stress resultants acting on the plate are written as

$$
\begin{gathered}
N_{p x}=C\left[\frac{\partial u_{p}}{\partial x}+v_{p} \frac{\partial v_{p}}{\partial x}+\frac{1}{2}\left(\frac{\partial w_{p}}{\partial x}\right)^{2}+\frac{1}{2} v_{p}\left(\frac{\partial w_{p}}{\partial y}\right)^{2}\right] \\
N_{p y}=C\left[\frac{\partial v_{p}}{\partial x}+v_{p} \frac{\partial u_{p}}{\partial x}+\frac{1}{2}\left(\frac{\partial w_{p}}{\partial y}\right)^{2}+\frac{1}{2} v_{p}\left(\frac{\partial w_{p}}{\partial x}\right)^{2}\right] \\
N_{p x y}=C \frac{1-v_{p}}{2}\left(\frac{\partial u_{p}}{\partial y}+\frac{\partial v_{p}}{\partial x}+\frac{\partial w_{p}}{\partial y} \frac{\partial w_{p}}{\partial x}\right) \\
M_{p x}=-D\left(\frac{\partial^{2} w_{p}}{\partial x^{2}}+v_{p} \frac{\partial^{2} w_{p}}{\partial y^{2}}\right) \\
M_{p y}=-D\left(\frac{\partial^{2} w_{p}}{\partial y^{2}}+v_{p} \frac{\partial^{2} w_{p}}{\partial x^{2}}\right) \\
M_{p x y}=-D\left(1-v_{p}\right) \frac{\partial^{2} w_{p}}{\partial x \partial y}
\end{gathered}
$$

where $C=E_{p} h_{p} /\left(1-v_{p}^{2}\right), D=E_{p} h_{p}^{3} / 12\left(1-v_{p}^{2}\right)$ are the membrane and bending rigidities of the plate, respectively.

On the basis of Hamilton's principle, the system of differential equations of motion of the plate can be expressed in terms of the displacement components as

$$
\begin{aligned}
& G_{p} h_{p}\left[\nabla^{2} u_{p}+\frac{1+v_{p}}{1-v_{p}} \frac{\partial}{\partial x}\left(\frac{\partial u_{p}}{\partial x}+\frac{\partial v_{p}}{\partial y}\right)+\left(\frac{2}{1-v_{p}} \frac{\partial^{2} w_{p}}{\partial x^{2}}+\frac{\partial^{2} w_{p}}{\partial y^{2}}\right) \frac{\partial w_{p}}{\partial x}+\frac{1+v_{p}}{1-v_{p}} \frac{\partial^{2} w_{p}}{\partial x \partial y} \frac{\partial w_{p}}{\partial y}\right]- \\
& -\rho_{p} h_{p} \ddot{u}_{p}=\sum_{i=1}^{I}\left(\sum_{j=1}^{2} q_{x j}^{i} \delta\left(y-y_{j}\right)\right)
\end{aligned}
$$




$$
\begin{aligned}
& G_{p} h_{p}\left[\nabla^{2} v_{p}+\frac{1+v_{p}}{1-v_{p}} \frac{\partial}{\partial y}\left(\frac{\partial u_{p}}{\partial x}+\frac{\partial v_{p}}{\partial y}\right)+\left(\frac{2}{1-v_{p}} \frac{\partial^{2} w_{p}}{\partial y^{2}}+\frac{\partial^{2} w_{p}}{\partial x^{2}}\right) \frac{\partial w_{p}}{\partial y}+\frac{1+v_{p}}{1-v_{p}} \frac{\partial^{2} w_{p}}{\partial x \partial y} \frac{\partial w_{p}}{\partial x}\right]- \\
& -\rho_{p} h_{p} \ddot{v}_{p}=\sum_{i=1}^{I}\left(\sum_{j=1}^{2} q_{y j}^{i} \delta\left(y-y_{j}\right)\right) \\
& D \nabla^{4} w_{p}-C\left\{\left[\left(\frac{\partial u_{p}}{\partial x}+\frac{1}{2}\left(\frac{\partial w_{p}}{\partial x}\right)^{2}\right)+v_{p}\left(\frac{\partial v_{p}}{\partial y}+\frac{1}{2}\left(\frac{\partial w_{p}}{\partial y}\right)^{2}\right)\right] \frac{\partial^{2} w_{p}}{\partial x^{2}}+\left(1-v_{p}\right) .\right. \\
& \cdot\left(\frac{\partial u_{p}}{\partial y}+\frac{\partial v_{p}}{\partial x}+\frac{\partial w_{p}}{\partial x} \frac{\partial w_{p}}{\partial y}\right) \frac{\partial^{2} w_{p}}{\partial x \partial y}+\left[\left(\frac{\partial v_{p}}{\partial y}+\frac{1}{2}\left(\frac{\partial w_{p}}{\partial y}\right)^{2}\right)+v_{p}\left(\frac{\partial u_{p}}{\partial x}+\frac{1}{2}\left(\frac{\partial w_{p}}{\partial x}\right)^{2}\right)\right] . \\
& \left.\cdot \frac{\partial^{2} w_{p}}{\partial y^{2}}\right\}+\rho_{p} h_{p} \ddot{w}_{p}-\rho_{p} h_{p} \ddot{u}_{p} \frac{\partial w_{p}}{\partial x}-\rho_{p} h_{p} \ddot{v}_{p} \frac{\partial w_{p}}{\partial y}-\frac{\rho_{p} h_{p}^{3}}{12} \frac{\partial^{2} \ddot{w}_{p}}{\partial x^{2}}-\frac{\rho_{p} h_{p}^{3}}{12} \frac{\partial^{2} \ddot{w}_{p}}{\partial y^{2}}= \\
& =g-\sum_{i=1}^{I}\left[\sum_{j=1}^{2}\left(q_{z j}^{i}+\frac{\partial m_{p x j}^{i}}{\partial y}+\frac{\partial m_{p y j}^{i}}{\partial x}-q_{x j}^{i} \frac{\partial w_{p j}^{i}}{\partial x}-q_{y j}^{i} \frac{\partial w_{p j}^{i}}{\partial y}\right) \delta\left(y-y_{j}\right)\right]
\end{aligned}
$$

where $\delta\left(y-y_{i}\right)$ is the Dirac's delta function in the $y$ direction.

The governing differential equations (4a)-(4c) are also subjected to the pertinent boundary conditions of the problem at hand

$$
\begin{gathered}
a_{p 1} u_{p n}+a_{p 2} N_{p n}=a_{p 3} \\
\beta_{p 1} u_{p t}+\beta_{p 2} N_{p t}=\beta_{p 3} \\
\gamma_{p 1} w_{p}+\gamma_{p 2} R_{p n}=\gamma_{p 3} \\
\delta_{p 1} \frac{\partial w_{p}}{\partial n}+\delta_{p 2} M_{p n}=\delta_{p 3} \\
\varepsilon_{1 k} w_{p}+\varepsilon_{2 k}\left\|T w_{p}\right\|_{k}=\varepsilon_{3 k}, \quad \varepsilon_{2 k} \neq 0
\end{gathered}
$$

and to the initial conditions

$$
\begin{array}{r}
w_{p}(\mathbf{x}, 0)=w_{p 0}(\mathbf{x}) \\
\dot{w}_{p}(\mathbf{x}, 0)=\bar{w}_{p 0}(\mathbf{x})
\end{array}
$$

where $a_{p l}, \beta_{p l}, \gamma_{p l}, \delta_{p l} \quad(l=1,2,3)$ are functions specified at the boundary $\Gamma$; $\varepsilon_{l k}(l=1,2,3)$ are functions specified at the $k$ corners of the plate; $w_{p 0}(\mathbf{x}), \bar{w}_{p 0}(\mathbf{x}), \mathbf{x}:\{x, y\}$ are the initial deflection and velocity of the points of the middle surface of the plate; $u_{p n}, u_{p t}$ and $N_{p n}, N_{p t}$ are the boundary membrane displacements and forces in the normal and tangential directions to the boundary, respectively; $R_{p n}$ and $M_{p n}$ are the effective reaction along the boundary and the bending moment normal to it, respectively, which employing intrinsic 
coordinates (i.e. the distance along the outward normal $n$ to the boundary and the arc length $s)$ are written as

$$
\begin{gathered}
R_{p n}=-D\left[\frac{\partial}{\partial n} \nabla^{2} w_{p}-\left(v_{p}-1\right) \frac{\partial}{\partial s}\left(\frac{\partial^{2} w_{p}}{\partial s \partial n}-\kappa \frac{\partial w_{p}}{\partial s}\right)\right]+N_{p n} \frac{\partial w_{p}}{\partial n}+N_{p t} \frac{\partial w_{p}}{\partial s} \\
M_{p n}=-D\left[\nabla^{2} w_{p}+\left(v_{p}-1\right)\left(\frac{\partial^{2} w_{p}}{\partial s^{2}}+\kappa \frac{\partial w_{p}}{\partial n}\right)\right]
\end{gathered}
$$

in which $\kappa(s)$ is the curvature of the boundary. Finally, $\left\|T w_{p}\right\|_{k}$ is the discontinuity jump of the twisting moment $T w_{p}$ at the corner $k$ of the plate, while $T w_{p}$ along the boundary is given by the following relation

$$
T w_{p}=D\left(v_{p}-1\right)\left(\frac{\partial^{2} w_{p}}{\partial s \partial n}-\kappa \frac{\partial w_{p}}{\partial s}\right)
$$

The boundary conditions (5a)-(5d) are the most general boundary conditions for the plate problem including also elastic support, while the corner condition (5e) holds for free or transversely elastically restrained corners $k$. It is apparent that all types of the conventional boundary conditions can be derived from these equations by specifying appropriately the functions $a_{p l}, \beta_{p l}, \gamma_{p l}$ and $\delta_{p l}(l=1,2,3)$ (e.g. for a clamped edge it is $\left.a_{p 1}=\beta_{p 1}=\gamma_{p 1}=\delta_{p 1}=1, a_{p 2}=a_{p 3}=\beta_{p 2}=\beta_{p 3}==\gamma_{p 2}=\gamma_{p 3}=\delta_{p 2}=\delta_{p 3}=0\right)$.

\section{b. For each (i-th) beam.}

Each beam undergoes transverse deflection with respect to $z^{i}$ and $y^{i}$ axes, as well as axial deformation and nonuniform angle of twist along $x^{i}$ axis. Based on the Bernoulli theory, the displacement field of an arbitrary point of a cross section (taking into account moderate large displacements and considering the angle of rotation of twist to have relatively small values) can be derived with respect to those of its centroid as

$$
\begin{aligned}
\bar{u}_{b}^{i}\left(x^{i}, y^{i}, z^{i}, t\right)= & u_{b}^{i}\left(x^{i}, t\right)-y^{i} \theta_{b z}^{i}\left(x^{i}, t\right)+z^{i} \theta_{b y}^{i}\left(x^{i}, t\right)+\frac{\partial \theta_{b x}^{i}}{\partial x^{i}} \varphi_{S}^{P i}\left(y^{i}, z^{i}, t\right) \\
& \bar{v}_{b}^{i}\left(x^{i}, y^{i}, z^{i}, t\right)=v_{b}^{i}\left(x^{i}, t\right)-z^{i} \theta_{b x}^{i}\left(x^{i}, t\right) \\
& \bar{w}_{b}^{i}\left(x^{i}, y^{i}, z^{i}, t\right)=w_{b}^{i}\left(x^{i}, t\right)+y^{i} \theta_{b x}^{i}\left(x^{i}, t\right) \\
\theta_{b y}^{i}\left(x^{i}, t\right)= & -\frac{\partial w_{b}^{i}\left(x^{i}, t\right)}{\partial x^{i}} \quad \theta_{b z}^{i}\left(x^{i}, t\right)=\frac{\partial v_{b}^{i}\left(x^{i}, t\right)}{\partial x^{i}}
\end{aligned}
$$

where $\bar{u}_{b}^{i}, \bar{v}_{b}^{i}, \bar{w}_{b}^{i}$ are the axial and transverse displacement components with respect to the $C^{i} x^{i} y^{i} z^{i}$ system of axes; $u_{b}^{i}=u_{b}^{i}\left(x^{i}\right), v_{b}^{i}=v_{b}^{i}\left(x^{i}\right)$ and $w_{b}^{i}=w_{b}^{i}\left(x^{i}\right)$ are the corresponding components of the centroid $C^{i} ; \theta_{b y}^{i}=\theta_{b y}^{i}\left(x^{i}\right), \theta_{b z}^{i}=\theta_{b z}^{i}\left(x^{i}\right)$ are the angles of rotation of the 
cross section due to bending, with respect to its centroid; $\partial \theta_{b x}^{i} / d x^{i}$ denotes the rate of change of the angle of twist $\theta_{b x}^{i}\left(x^{i}\right)$ regarded as the torsional curvature and $\varphi_{S}^{P i}$ is the primary warping function with respect to the cross section's shear center (coinciding with its centroid). Employing again the strain-displacement relations of the three-dimensional elasticity for moderate displacements [4], the Hooke's stress-strain law and integrating the arising stress components over the beam's cross section after ignoring the nonlinear terms with respect to the angle of twist and its derivatives, the stress resultants of the beam are derived as

$$
\begin{gathered}
N_{b}^{i}=E_{b}^{i} A_{b}^{i}\left[\frac{\partial u_{b}^{i}}{\partial x^{i}}+\frac{1}{2}\left(\left(\frac{\partial v_{b}^{i}}{\partial x^{i}}\right)^{2}+\left(\frac{\partial w_{b}^{i}}{\partial x^{i}}\right)^{2}\right)\right] \\
M_{b y}^{i}=-E_{b}^{i} I_{y}^{i} \frac{\partial^{2} w_{b}^{i}}{\partial x^{i 2}} \\
M_{b z}^{i}=E_{b}^{i} I_{z}^{i} \frac{\partial^{2} v_{b}^{i}}{\partial x^{i 2}} \\
M_{b t}^{P i}=G_{b}^{i} I_{t}^{i} \frac{\partial \theta_{b x}^{i}}{\partial x^{i}} \\
M_{b w}^{i}=-E_{b}^{i} C_{S}^{i} \frac{\partial^{2} \theta_{b x}^{i}}{\partial x^{i 2}}
\end{gathered}
$$

where $M_{b t}^{P i}$ is the primary twisting moment [5] resulting from the primary shear stress distribution; $M_{b w}^{i}$ is the warping moment due to torsional curvature. Furthermore $I_{y}^{i}, I_{z}^{i}$ are the principal moments of inertia; $I_{S}^{i}$ is the polar moment of inertia, while $I_{t}^{i}$ and $C_{S}^{i}$ are the torsion and warping constants of the i-th beam with respect to the cross section's shear center (coinciding with its centroid), respectively, given as [6]

$$
\begin{gathered}
I_{t}^{i}=\int_{\Omega}\left(y^{i 2}+z^{i 2}+y^{i} \frac{\partial \varphi_{S}^{P i}}{\partial z^{i}}-z^{i} \frac{\partial \varphi_{S}^{P i}}{\partial y^{i}}\right) d \Omega \\
C_{S}^{i}=\int_{\Omega}\left(\varphi_{S}^{P i}\right)^{2} d \Omega
\end{gathered}
$$

On the basis of Hamilton's principle, the differential equations of motion are obtained as

$$
\begin{gathered}
-E_{b}^{i} A_{b}^{i}\left(\frac{\partial^{2} u_{b}^{i}}{\partial x^{i 2}}+\frac{\partial w_{b}^{i}}{\partial x^{i}} \frac{\partial^{2} w_{b}^{i}}{\partial x^{i 2}}+\frac{\partial v_{b}^{i}}{\partial x^{i}} \frac{\partial^{2} v_{b}^{i}}{\partial x^{i 2}}\right)+\rho_{b}^{i} \mathrm{~A}_{b}^{i} \ddot{u}_{b}^{i}=\sum_{j=1}^{2} q_{x j}^{i} \\
E_{b}^{i} I_{z}^{i} \frac{\partial^{4} v_{b}^{i}}{\partial x^{i 4}}-N_{b}^{i} \frac{\partial^{2} v_{b}^{i}}{\partial x^{i 2}}-\rho_{b}^{i} I_{z}^{i} \frac{\partial^{2} \ddot{v}_{b}}{\partial x^{2}}+\rho_{b}^{i} \mathrm{~A}_{b}^{i} \ddot{v}_{b}-\rho_{b}^{i} \mathrm{~A}_{b}^{i} \ddot{u}_{b}^{i} \frac{\partial v_{b}^{i}}{\partial x^{i}}=\sum_{j=1}^{2}\left(q_{y j}^{i}-q_{x j}^{i} \frac{\partial v_{b}^{i}}{\partial x^{i}}-\frac{\partial m_{b z j}^{i}}{\partial x^{i}}\right) \\
E_{b}^{i} I_{y}^{i} \frac{\partial^{4} w_{b}^{i}}{\partial x^{i 4}}-N_{b}^{i} \frac{\partial^{2} w_{b}^{i}}{\partial x^{i 2}}-\rho_{b}^{i} I_{y}^{i} \frac{\partial^{2} \ddot{w}_{b}}{\partial x^{2}}+\rho_{b}^{i} \mathrm{~A}_{b}^{i} \ddot{w}_{b}-\rho_{b}^{i} \mathrm{~A}_{b}^{i} \ddot{u}_{b}^{i} \frac{\partial w_{b}^{i}}{\partial x^{i}}=\sum_{j=1}^{2}\left(q_{z j}^{i}-q_{x j}^{i} \frac{\partial w_{b}^{i}}{\partial x^{i}}+\frac{\partial m_{b y j}^{i}}{\partial x^{i}}\right)
\end{gathered}
$$




$$
E_{b}^{i} C_{S}^{i} \frac{\partial^{4} \theta_{b x}^{i}}{\partial x^{i 4}}-G_{b}^{i} I_{t}^{i} \frac{\partial^{2} \theta_{b x}^{i}}{\partial x^{i 2}}+\rho_{b}^{i} I_{S}^{i} \ddot{\theta}_{b x}^{i}-\rho_{b}^{i} C_{S}^{i} \frac{\partial^{2} \ddot{\theta}_{b x}^{i}}{\partial x^{2}}=\sum_{j=1}^{2}\left[m_{b x j}^{i}+\frac{\partial m_{b w j}^{i}}{\partial x^{i}}\right]
$$

Moreover, the corresponding boundary conditions of the i-th beam at its ends $x^{i}=0, l^{i}$ are given as

$$
\begin{array}{cc}
\beta_{b 1}^{i} v_{b}^{i}+\beta_{b 2}^{i} R_{b y}^{i}=\beta_{b 3}^{i}+\alpha_{b 2}^{i} N_{b}^{i}=\alpha_{b 3}^{i} \\
\gamma_{b 1}^{i} w_{b}^{i}+\gamma_{b 2}^{i} R_{b z}^{i}=\gamma_{b 3}^{i} & \bar{\beta}_{b 1}^{i} \theta_{b z}^{i}+\bar{\beta}_{b 2}^{i} M_{b z}^{i}=\bar{\beta}_{b 3}^{i} \\
\delta_{b 1}^{i} \theta_{b x}^{i}+\delta_{b 2}^{i} M_{b t}^{i}=\delta_{b 3}^{i} & \bar{\gamma}_{b 1}^{i} \theta_{b y}^{i}+\bar{\gamma}_{b 2}^{i} M_{b y}^{i}=\bar{\gamma}_{b 3}^{i} \\
\bar{\delta}_{b 1}^{i} \frac{d \theta_{b x}^{i}}{d x}+\bar{\delta}_{b 2}^{i} M_{b w}^{i}=\bar{\delta}_{b 3}^{i}
\end{array}
$$

and the initial conditions as

$$
\begin{aligned}
& w_{b}^{i}\left(x^{i}, 0\right)=w_{b 0}^{i}\left(x^{i}\right) \\
& \dot{w}_{b}^{i}\left(x^{i}, 0\right)=\bar{w}_{b 0}^{i}\left(x^{i}\right)
\end{aligned}
$$

where the angles of rotation of the cross section due to bending $\theta_{b y}^{i}, \theta_{b z}^{i}$ are given from eqns. (9d,e), $R_{b y}^{i}, R_{b z}^{i}$ and $M_{b z}^{i}, M_{b y}^{i}$ are the reactions and bending moments with respect to $y^{i}$, $z^{i}$ axes, respectively, which after applying the aforementioned simplifications are given as

$$
\begin{gathered}
R_{b y}^{i}=N_{b}^{i} \frac{\partial v_{b}^{i}}{\partial x^{i}}-E_{b}^{i} I_{z}^{i} \frac{\partial^{3} v_{b}^{i}}{\partial x^{i 3}} \\
R_{b z}^{i}=N_{b}^{i} \frac{\partial w_{b}^{i}}{\partial x^{i}}-E_{b}^{i} I_{y}^{i} \frac{\partial^{3} w_{b}^{i}}{\partial x^{i 3}} \\
M_{b z}^{i}=E_{b}^{i} I_{z}^{i} \frac{\partial^{2} v_{b}^{i}}{\partial x^{i 2}} \\
M_{b y}^{i}=-E_{b}^{i} I_{y}^{i} \frac{\partial^{2} w_{b}^{i}}{\partial x^{i 2}}
\end{gathered}
$$

and $M_{b t}^{i}, M_{b w}^{i}$ are the torsional and warping moments at the boundaries of the beam, respectively, given as

$$
\begin{gathered}
M_{b t}^{i}=G_{b}^{i} I_{t}^{i} \frac{\partial \theta_{b x}^{i}}{\partial x^{i}}-E_{b}^{i} C_{S}^{i} \frac{\partial^{3} \theta_{b x}^{i}}{\partial x^{i 3}} \\
M_{b w}^{i}=-E_{b}^{i} C_{S}^{i} \frac{\partial^{2} \theta_{b x}^{i}}{\partial x^{i 2}}
\end{gathered}
$$

Finally, $\alpha_{b k}^{i}, \beta_{b k}^{i}, \bar{\beta}_{b k}^{i}, \gamma_{b k}^{i}, \bar{\gamma}_{b k}^{i}, \delta_{b k}^{i}, \bar{\delta}_{b k}^{i}(k=1,2,3)$ are functions specified at the i-th beam ends $\left(x^{i}=0, l^{i}\right)$. The boundary conditions (13)-(16a,b) are the most general boundary conditions for the beam problem including also the elastic support. It is apparent that all types 
of the conventional boundary conditions (clamped, simply supported, free or guided edge) can be derived from these equations by specifying appropriately the aforementioned coefficients.

Eqns. (4), (12) constitute a set of seven coupled and nonlinear partial differential equations including thirteen unknowns, namely $u_{p}, v_{p}, w_{p}, u_{b}^{i}, v_{b}^{i}, w_{b}^{i}, \theta_{b x}^{i}, q_{x 1}^{i}, q_{y 1}^{i}, q_{z 1}^{i}, q_{x 2}^{i}, q_{y 2}^{i}, q_{z 2}^{i}$. Six additional equations are required, which result from the displacement continuity conditions in the directions of $x^{i}, y^{i}$ and $z^{i}$ local axes along the two interface lines of each (i-th) plate - beam interface. Taking into account the displacement fields expressed by eqns. (1a)(1c) and (9a)-(9d,e) the displacement continuity conditions [7] can be expressed as

In the direction of $x^{i}$ local axis:

$$
\begin{gathered}
u_{p 1}^{i}-u_{b}^{i}=\frac{h_{p}}{2} \frac{\partial w_{p 1}^{i}}{\partial x}+\frac{h_{b}^{i}}{2} \frac{\partial w_{b}^{i}}{\partial x^{i}}+\frac{b_{f}^{i}}{4} \frac{\partial v_{b}^{i}}{\partial x^{i}}+\frac{\partial \theta_{b x}^{i}}{\partial x^{i}}\left(\varphi_{S}^{P i}\right)_{f 1}+\frac{q_{x 1}^{i}}{k_{x 1}^{i}}\left[1-\frac{1}{2}\left(\frac{\partial w_{b}^{i}}{\partial x^{i}}\right)^{2}\right] \\
\text { along interface line } 1\left(f_{j=1}^{i}\right) \\
u_{p 2}^{i}-u_{b}^{i}=\frac{h_{p}}{2} \frac{\partial w_{p 2}^{i}}{\partial x}+\frac{h_{b}^{i}}{2} \frac{\partial w_{b}^{i}}{\partial x^{i}}-\frac{b_{f}^{i}}{4} \frac{\partial v_{b}^{i}}{\partial x^{i}}+\frac{\partial \theta_{b x}^{i}}{\partial x^{i}}\left(\varphi_{S}^{P i}\right)_{f 2}+\frac{q_{x 2}^{i}}{k_{x 2}^{i}}\left[1-\frac{1}{2}\left(\frac{\partial w_{b}^{i}}{\partial x^{i}}\right)^{2}\right] \\
\text { along interface line } 2\left(f_{j=2}^{i}\right)
\end{gathered}
$$

In the direction of $y^{i}$ local axis:

$$
\begin{aligned}
& v_{p 1}^{i}-v_{b}^{i}=\frac{h_{p}}{2} \frac{\partial w_{p 1}^{i}}{\partial y}+\frac{h_{b}^{i}}{2} \theta_{b x}^{i}+\frac{q_{y 1}^{i}}{k_{y 1}^{i}}\left[1-\frac{1}{2}\left(\theta_{b x}^{i}\right)^{2}\right] \quad \text { along interface line } 1\left(f_{j=1}^{i}\right) \\
& v_{p 2}^{i}-v_{b}^{i}=\frac{h_{p}}{2} \frac{\partial w_{p 2}^{i}}{\partial y}+\frac{h_{b}^{i}}{2} \theta_{b x}^{i}+\frac{q_{y 2}^{i}}{k_{y 2}^{i}}\left[1-\frac{1}{2}\left(\theta_{b x}^{i}\right)^{2}\right] \quad \text { along interface line } 2\left(f_{j=2}^{i}\right)
\end{aligned}
$$

In the direction of $z^{i}$ local axis:

$$
\begin{array}{ll}
w_{p 1}^{i}-w_{b}^{i}=-\frac{b_{f}^{i}}{4} \theta_{b x}^{i} & \text { along interface line } 1\left(f_{j=1}^{i}\right) \\
w_{p 2}^{i}-w_{b}^{i}=\frac{b_{f}^{i}}{4} \theta_{b x}^{i} & \text { along interface line } 2\left(f_{j=2}^{i}\right)
\end{array}
$$

where $\left(\varphi_{S}^{P i}\right)_{f j}$ is the value of the primary warping function with respect to the shear center of the beam cross section (coinciding with its centroid) at the point of the j-th interface line of the i-th plate - beam interface $f_{j}^{i}$ and $k_{x j}^{i}, k_{y j}^{i}$ are the stiffness of the arbitrarily distributed shear connectors along the $x^{i}$ and $y^{i}$ directions, respectively. It is noted that $k_{x j}^{i}=k_{x j}^{i}\left(s_{x j}^{i}\right)$ and $k_{y j}^{i}=k_{y j}^{i}\left(s_{y j}^{i}\right)$ can represent any linear or nonlinear relationship between the in-plane in- 
terface forces and the interface slip $s_{j}^{i}$ in the corresponding direction. In all of the aforementioned equations the values of the primary warping function $\varphi_{S}^{P i}\left(y^{i}, z^{i}\right)$ should be set having the appropriate algebraic sign corresponding to the local beam axes.

\section{INTEGRAL REPRESENTATIONS - NUMERICAL SOLUTION}

The solution of the presented dynamic problem requires the integration of the set of Eqs. (4a)-(4c), (12a)-(12d) subjected to the prescribed boundary and initial conditions. Moreover, the displacement continuity conditions should also be fulfilled. Due to the nonlinear and coupling character of the equations of motion, an analytical solution is out of question. Therefore, a numerical solution is derived employing the Analog Equation Method [2], a BEM based method. Contrary to previous research efforts where the numerical analysis is based on BEM using a lumped mass assumption model after evaluating the flexibility matrix at the mass nodal points [5], in this work a distributed mass model is employed.

\section{NUMERICAL EXAMPLES}

On the basis of the analytical and numerical procedures presented in the previous sections, a FORTRAN program has been written and representative examples have been studied to demonstrate the effectiveness, wherever possible the accuracy and the range of applications of the proposed method. It is noted that the term "linear analysis" appearing in all the following sections, refers to the solution of the previously obtained system of equations neglecting all of the nonlinear terms.

\section{Example 1}

A rectangular plate $\left(h_{p}=0.2 \mathrm{~cm}, E_{p}=E_{b}=3 \times 10^{7} \mathrm{kN} / \mathrm{m}^{2}, \rho_{p}=\rho_{b}=2.5 \mathrm{tn} / \mathrm{m}^{3}\right.$, $v_{p}=v_{b}=0.2$ ) with dimensions $L_{x} \times L_{y}=18 \mathrm{~cm} \times 9 \mathrm{~cm}$ subjected to a suddenly applied uniform load $g=40 \mathrm{kN} / \mathrm{m}^{2}$ and stiffened by a rectangular beam of $0.5 \mathrm{~cm}$ height and $1.0 \mathrm{~cm}$ width, eccentrically placed with respect to the plate centre line, has been studied. The plate is clamped along its small edges, while the rest of the edges are free according to the transverse and in-plane boundary conditions. The connection between the slab and the beam was accomplished using a linear distribution of shear connectors along each interface (Figure 4). The adopted relationship for the shear connectors' stiffness is given as

$$
k_{x j}^{i}=k_{y j}^{i}=250 K\left|x^{i}-\frac{l_{b x}^{i}}{2}\right| k N / m^{2} \quad(j=1,2)
$$

where $\mathrm{K}$ is dimensionless magnification factor. In Table 1 the obtained maximum deflections $w_{p}$ of the first cycle of motion of the stiffened plate, at the middle of the free edge A are shown for various values of K. In Figure 5, the time histories of the deflection $w_{p}(t)$ at the same point $\mathrm{A}$ for the cases of linear and nonlinear dynamic analysis, for various values of $\mathrm{K}$, are presented. Moreover, in Figure 6 the contour lines of the displacement $w_{p}$ of the stiffened plate for full and partial connection between plate and beam, at the time of maximum transverse displacement are shown, ignoring (Figure 6a,c) or taking into account (Figure 6b,d) geometric nonlinearities. 


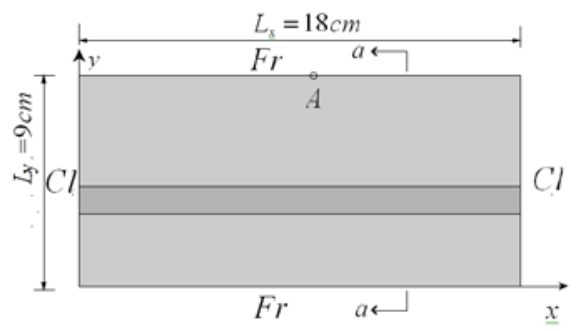

(a)

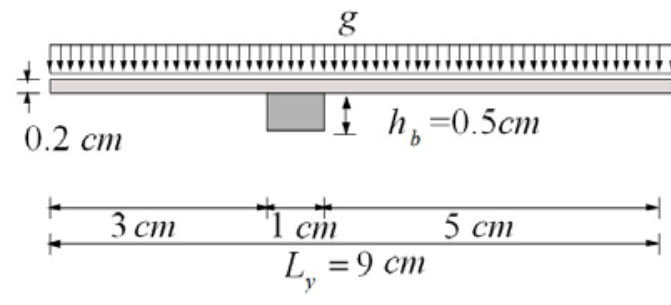

(b)

Figure 4: Plan view (a) and section a-a (b) of the stiffened plate of example 1.

\begin{tabular}{ccc}
\hline \hline$K$ & Linear Analysis & Nonlinear Analysis \\
\hline Full Connection & 0.431 & 0.402 \\
\hline 100.0 & 0.433 & 0.403 \\
\hline 10.0 & 0.437 & 0.406 \\
\hline 1.0 & 0.453 & 0.420 \\
\hline 0.1 & 0.489 & 0.452 \\
\hline 0.01 & 0.565 & 0.520 \\
\hline 0.005 & 0.585 & 0.530 \\
\hline \hline
\end{tabular}

Table 1: Maximum deflection $w_{p}(\mathrm{~m})$ at the middle point A of the first circle of motion of the stiffened plate for various values of $K$ of example 1 .

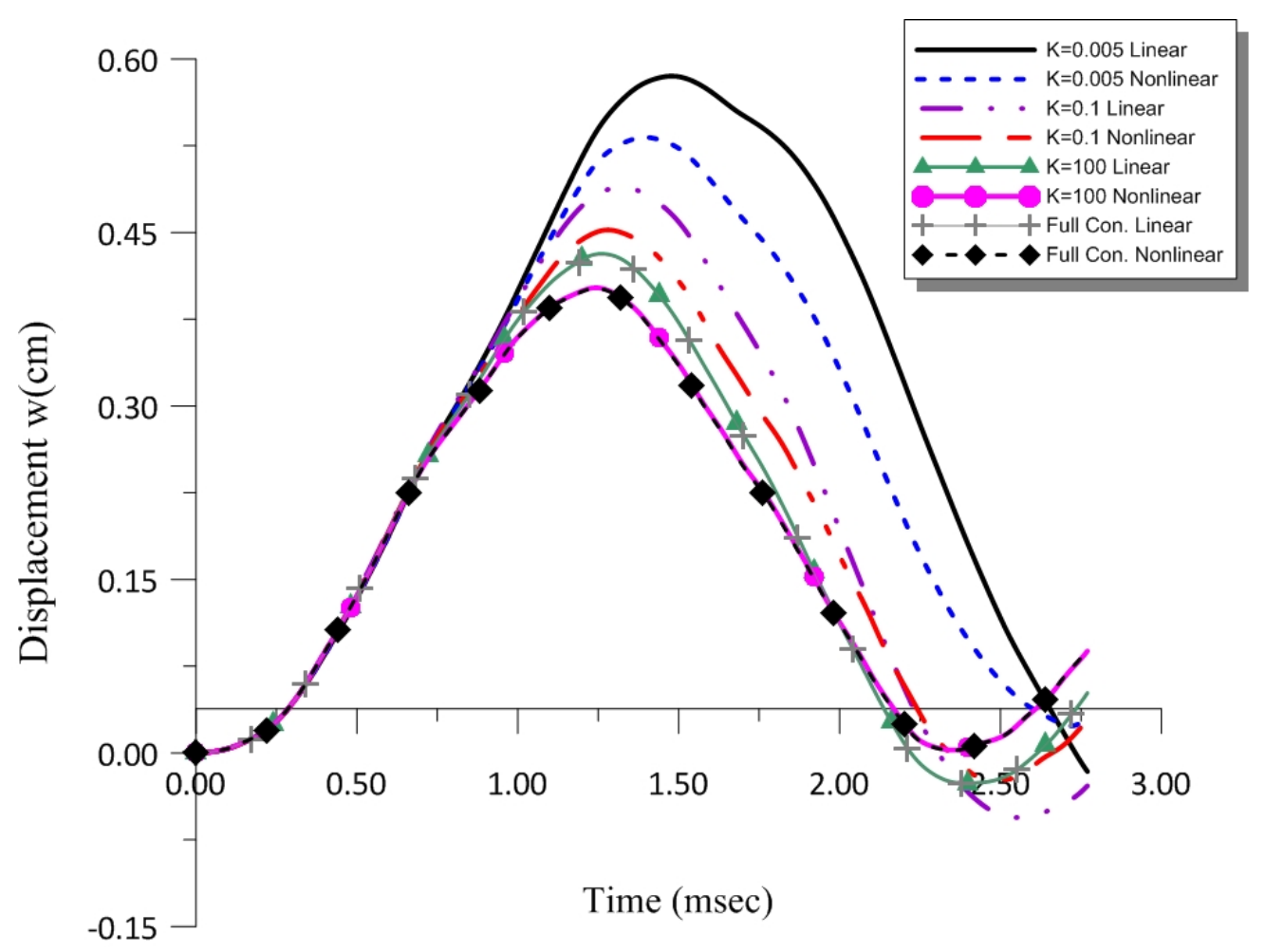

Figure 5: Time history of displacement $\mathrm{w}(\mathrm{cm})$ at the middle A of the free edge of the plate of example 1. 

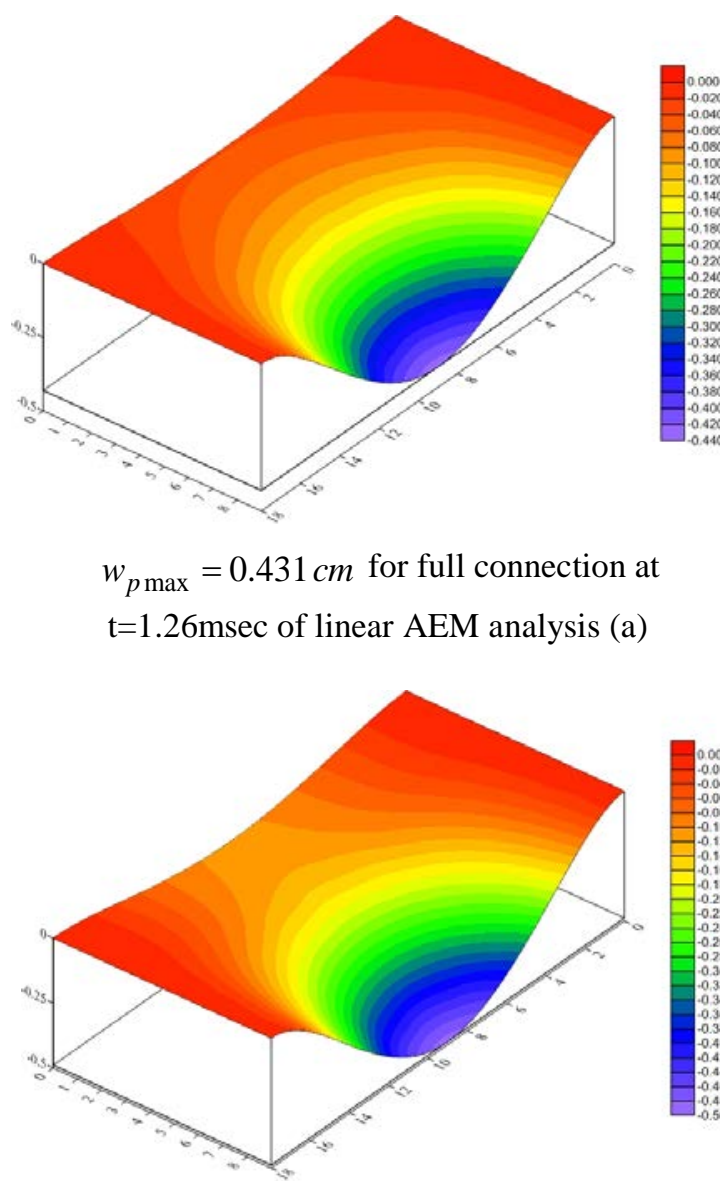

$w_{p \max }=0.489 \mathrm{~cm}$ for $\mathrm{K}=0.1$ at $\mathrm{t}=1.32 \mathrm{msec}$ of linear AEM analysis (c)

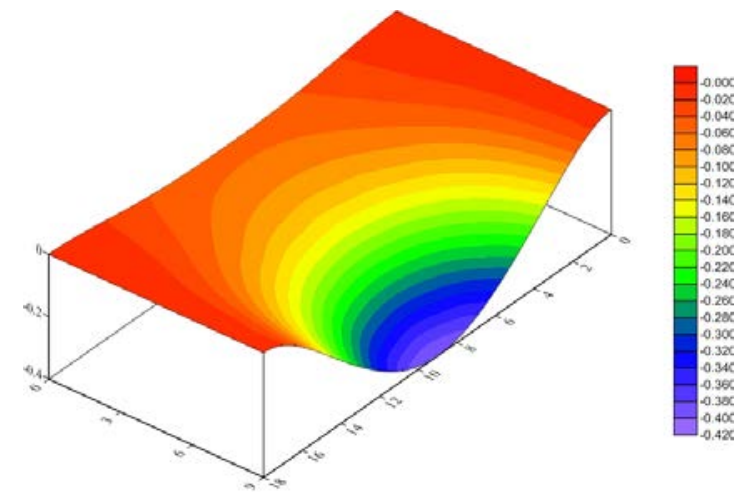

$$
w_{p \max }=0.402 \mathrm{~cm} \text { for full connection at }
$$

$\mathrm{t}=1.24 \mathrm{msec}$ of nonlinear AEM analysis (b)

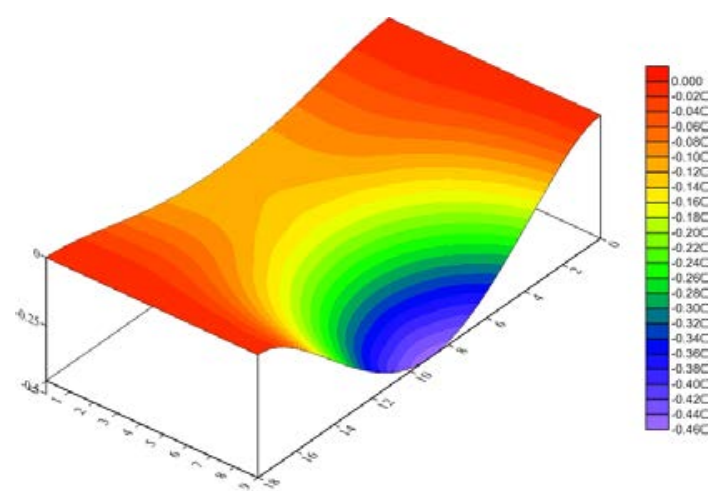

$w_{p \max }=0.452 \mathrm{~cm}$ for $\mathrm{K}=0.1$ at $\mathrm{t}=1.28 \mathrm{msec}$ of nonlinear AEM analysis (d)

Figure 6: Contour lines of $w_{p}$ of the stiffened plate of example 1, for full and partial connection between plate and beam, at the time of maximum transverse displacement ignoring $(a, c)$ or taking into account $(b, d)$ geometric nonlinearities.

\section{CONCLUDING REMARKS}

A general solution for the geometrically nonlinear dynamic analysis of plates stiffened by arbitrarily placed parallel beams of arbitrary doubly symmetric cross section with deformable connections, subjected to arbitrary loading is presented. The proposed model takes into account the nonuniform distribution of the interface shear forces and the nonuniform torsional response of the beams. The main conclusions that can be drawn from this investigation are

- The proposed model permits the study of the dynamic response of stiffened plates subjected to arbitrary loading, while both the number and the placement of the parallel beams are also arbitrary (eccentric beams are included). The plate and the beams are supported by the most general boundary conditions including elastic support or restraint.

- The adopted model permits the evaluation of the longitudinal and transverse in-plane shear forces at the interfaces between the plate and the beams in the geometrically non- 
linear dynamic analysis of the stiffened plate taking into account interface slip in both the $\mathrm{x}$ and $\mathrm{y}$ directions.

- The nonuniform torsion in which the stiffening beams are subjected is taken into account by solving the corresponding problem and by comprehending the arising twisting and warping in the corresponding displacement continuity conditions. The distributed warping moment arising from the nonuniform distribution of longitudinal in-plane forces is also taken into account.

- The influence of geometrical nonlinearity on the deformation of the examined stiffened plates is remarkable.

- The developed procedure retains most of the advantages of a BEM solution over a FEM approach, although it requires domain discretization.

- The increment of the deflection with the decrement of the connectors' stiffness is easily verified, while this decrement results in a more pronounced influence of the geometrical nonlinearity on the response of the stiffened plate.

\section{Acknowledgments}

This work has been funded by the State Scholarships Foundation of Greece as a part of postdoctoral research project.

\section{REFERENCES}

[1] E.J. Sapountzakis, V.G Mokos, An Improved Model for the Analysis of Plates Stiffened by Parallel Beams with Deformable Connection, Computers and Structures, 86, 21662181, 2008.

[2] J.T. Katsikadelis, The Analog Equation Method. A Boundary - only Integral Equation Method for Nonlinear Static and Dynamic Problems in General Bodies, Theoretical and Applied Mechanics, 27, 13-38, 2002.

[3] E.J. Sapountzakis, V.G. Mokos, Analysis of Plates Stiffened by Parallel Beams, International Journal for Numerical Methods in Engineering, 70, 1209-1240, 2007.

[4] E. Ramm, T.J. Hofmann, Stabtragwerke, Der Ingenieurbau, Ed.G. Mehlhorn, Band Baustatik/Baudynamik, Ernst \& Sohn, Berlin, 1995.

[5] E.J. Sapountzakis, V.G. Mokos, An Improved Model for the Dynamic Analysis of Plates Stiffened by Parallel Beams, Engineering Structures, 30, 1720-1733, 2008.

[6] E.J. Sapountzakis, V.G. Mokos, Warping Shear Stresses in Nonuniform Torsion by BEM, Computational Mechanics, 30, 131-142, 2003.

[7] E.J. Sapountzakis, I.C. Dikaros, Large Deflection Analysis of Plates Stiffened by Parallel Beams with Deformable Connection, Journal of Engineering Mechanics, ASCE, 138, (8), 1021-1041, 2012. 